\title{
Lifethreatening tracheal obstruction in a child caused by nodular fasciitis: case presentation
}

\author{
Yusuf Shieba $^{1^{*}} \mathbb{0}$, Mahmoud Khairy ${ }^{2}$, Mohamed Elzouk ${ }^{3}$ and Albaraa Ali Mansour ${ }^{4}$
}

\begin{abstract}
Background: Nodular fasciitis (NF) is a rare non-neoplastic inflammatory tumor and usually presents as a painless, rapidly growing subcutaneous soft tissue mass. The head and neck are relatively common locations for nodular fasciitis, particularly in children. NF in the trachea is rare and may evolve to a fatal condition, especially due to its rapidly growing nature that can cause life-threatening tracheal obstruction.

Case presentation: We report the case of a 5-year-old child with NF in the trachea and subtotal tracheal obstruction. Bronchoscopy and biopsy proved the diagnosis, and bronchoscopic excision of the tumor was performed.

Conclusions: NF is a rare airway tumor, occurring mostly in adults, and may presenting with pneumonia-like symptoms. Early detection of the lesion is essential to avoid life-threatening airway obstruction.
\end{abstract}

Keywords: Nodular fasciitis, Tracheal obstruction, Subcutaneous pseudosarcomatous fibromatosis

\section{Background}

$\mathrm{NF}$ is characterized as a non-malignant, proliferative reactive lesion containing fibroblasts and myofibroblasts [1]. It can generally be found in the extremities and in the trunk. NF prevalence is $13-20 \%$ in the head and neck area. The precise causes of the lesion are unclear at the time [2]. The research clearly shows that prior trauma is a critical etiological element, but it is not often depicted in studies. It represents as a solid mass $1-5 \mathrm{ml}$ in diameter. It may even spread through the tissue under the skin and muscles. Its occurrence rate is increasing, and is often misdiagnosed as malignant. NF needs to be diagnosed early [3].

\footnotetext{
${ }^{*}$ Correspondence: yusuf.shieba@med.svu.edu.eg

${ }^{1}$ Department of Cardiothoracic Surgery, Faculty of Medicine, South Valley

University, Safaga Road, Qena 83523, Egypt

Full list of author information is available at the end of the article
}

\section{Case presentation}

We report the case of a 5-year-old boy who was referred to our emergency department with severe respiratory distress, and the patient was free of srtidor. Four months before presentation, the child had signs of a respiratory infection, interpreted as pneumonia. He had been admitted to the pediatric department three times. His mother confirmed that he had not inhaled a foreign body, and there is also no history of hemoptysis.

Nevertheless, a computed tomography (CT) scan was performed to exclude a radiolucent foreign body. It showed a space-occupying lesion in the trachea $3.2 \mathrm{~cm}$ above the bifurcation measuring $4 \times 1.6 \mathrm{~cm}$ with a pneumonic consolidation in the right lower lobe (Fig. 1).

The patient was referred to our cardiothoracic surgery department and prepared for urgent rigid bronchoscopy performed under general anesthesia. It revealed a solitary soft tissue mass in the trachea with a subtotal obstruction. The mass appeared to be white, soft, circumscribed, vulnerable, and obstructed nearly the whole lumen of the trachea, leaving part of the lumen free. As a result of bleeding during the rigid bronchoscopy, the trachea was totally occluded. 


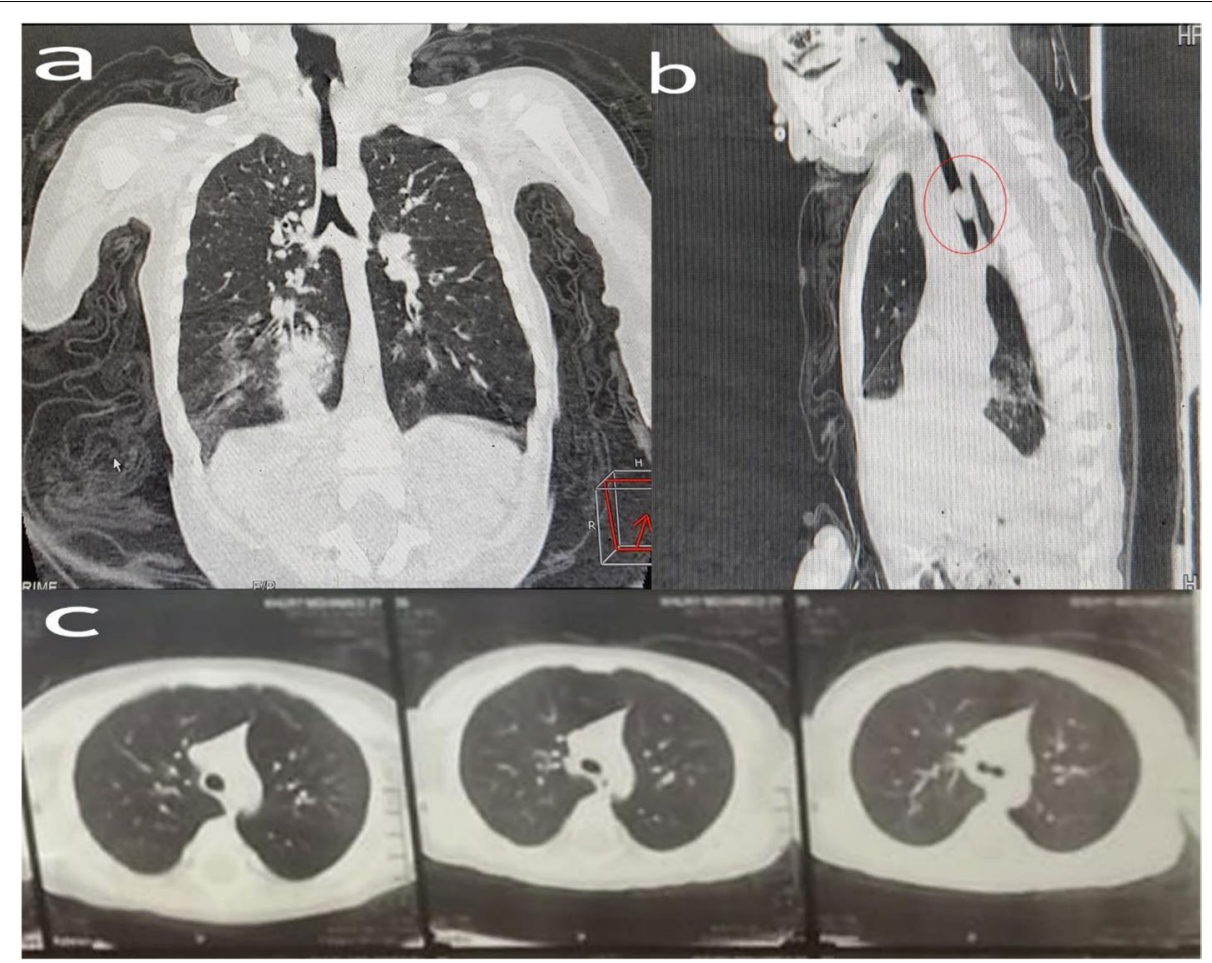

Fig. 1 CT scan showing mass in the trachea with a pneumonic patch in the RLL. a Coronal view b Sagittal view. c 6 months follow-up CT chest (cross-sectional view)

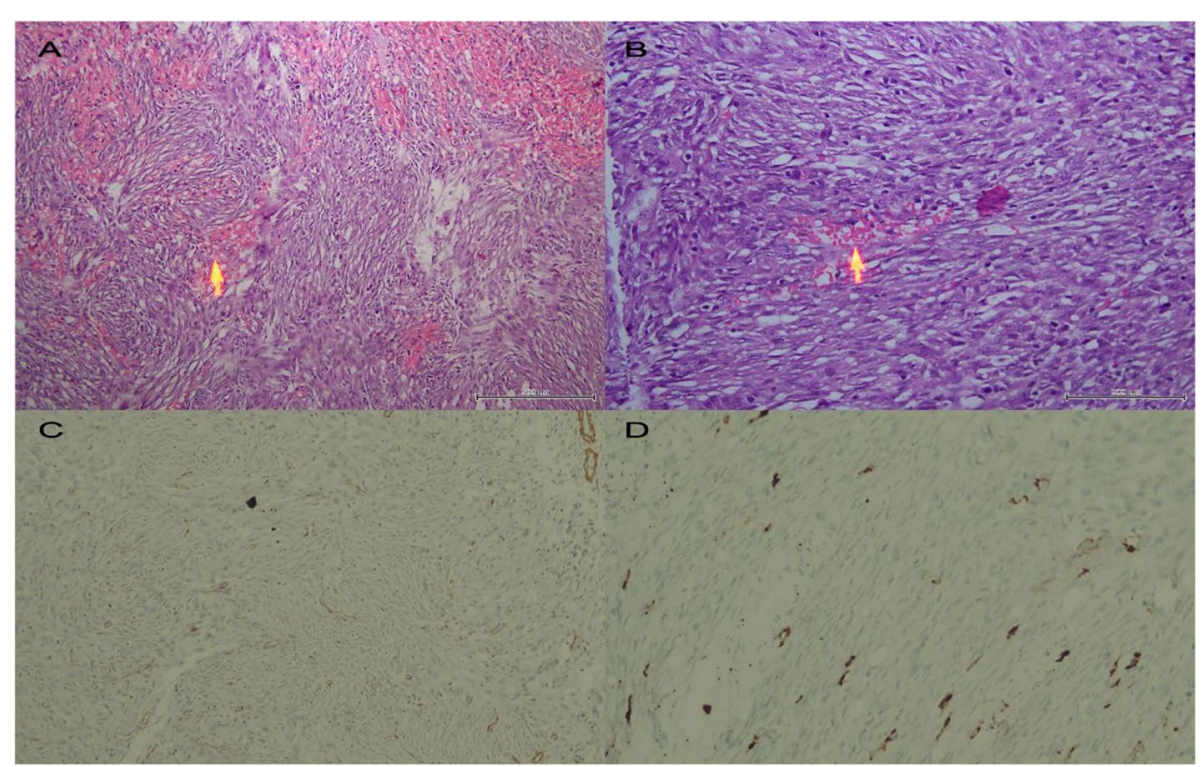

Fig. 2 A, B Cross section of soft tissue mass sample with different power of magnifications fibroblastic spindle cells arranged in a fascicular pattern. C SMA positive immunostaining of cells of nodular fasciitis. Magnification $=\times 100$. D CD10 positive immunostaining of cells of nodular fasciitis. Magnification $=\times 200$ 
This caused a hypoxic cardiac arrest for the duration of 1 min. The bleeding was controlled after applying a topical vasoconstrictor, and debulking of the tumor was performed, and a biopsy was sent for histopathological examination. The patient was transferred to the pediatric intensive care unit (PICU), and steroid therapy was administered. Histopathologic examination revealed fibroblastic spindle cells arranged in a fascicular pattern with scattered inflammatory cells, without malignant cells, SMA and $\mathrm{CD}_{10}$ stains were positive, confirming NF, while $\mathrm{P}_{53}$ was negative (Fig. 2).

The patient underwent rigid bronchoscopy under general anesthesia. The electrocautery was used to excise the tumor. The thermal effect degenerates proteins, thus inducing tissue necrosis. Thereafter, the necrotic tissue could be easily removed using a biopsy forceps. The postoperative period was uneventful and he stayed in the hospital for 3 days. The patient was followed six months after surgery with $\mathrm{CT}$ chest, which revealed no local recurrence of the tumor (Fig. 1).

\section{Discussion}

NF is a benign soft tissue inflammatory tumor composed of fibroblastic cells with myofibroblastic proliferation. It is also known as subcutaneous pseudosarcomatous fibromatosis [1]. The etiology is still unclear, but it might be caused by unusual myofibroblast proliferation preceded by an inflammatory proces, infection, or local injury [4]. Although NF may occur in patients of any age, it is most common in adults (20 to 40 years). Only $14 \%$ of the patients are $<10$ or $>60$ years old. The sex distribution is equal [5]. Although it may occur virtually anywhere on the body, most of the cases present with superficial soft tissue masses in the extremities or trunk.

Fabre et al. reported a high recurrence rate of pulmonary inflammatory pseudotumors that ranged from 18 to $40 \%$ and concluded that complete surgical resection had excellent long-term results [6].

The preferred treatment for NF is local excision. Recurrence is rare if totally excised as it has a good prognosis and metastases do not occur. Rare spontaneous regression and rapid resolution with intralesional corticosteroid injection in lesions that cannot be excised have been reported [7].

\section{Conclusion}

In conclusion, NF is a rare airway tumor, occurring mostly in adults, and may presenting with pneumonialike symptoms. Early detection of the lesion is essential to avoid life-threatening airway obstruction. Also, the complete surgical resection with termino-terminal anastomosis is the gold standard of treatment if feasible.

\section{Abbreviations}

CT: Computed tomography; NF: Nodular fasciitis; PICU: Pediatric intensive care unit; SMA: Smooth muscle actin.

\section{Acknowledgements}

We would like to express our gratitude to Dr. Mahmoud I. El Dosoky for his generous assistance in photographing slides in the pathology department. Large sections of this case report have been taken from the preprint version (DOI: 10.21203/rs.3.rs-94187/).

\section{Authors' contributions}

YS: writing, editing. MK: reviewing. ME: writing. AM: writing, editing. All authors read and approved the final manuscript.

\section{Funding}

The authors did not receive any financial support for this research.

\section{Availability of data and materials}

The datasets used or analyzed during the current study are available from the corresponding author on reasonable request.

\section{Declarations}

\section{Ethics approval and consent to participate}

This study protocol conforms to the ethical standards of the Helsinki Declaration and approval was obtained from Qena University Hospital, Faculty of Medicine, South Valley University. Committee's reference number is not available. Informed written consent to participate is obtained.

\section{Consent for publication}

Appropriate written informed consent was obtained for publication of this case report and accompanying image from patient parent.

\section{Competing interests}

The authors declare that they have no competing interests.

\section{Author details \\ 'Department of Cardiothoracic Surgery, Faculty of Medicine, South Valley University, Safaga Road, Qena 83523, Egypt. ${ }^{2}$ Department of Cardiothoracic Surgery, Faculty of Medicine, Assiut University, Assiut, Egypt. ${ }^{3}$ Department of Anesthesia, Faculty of Medicine, South Valley University, Qena, Egypt. ${ }^{4}$ Department of Cardiac Surgery, National Heart Institute, Cairo, Egypt.}

Received: 14 August 2021 Accepted: 2 January 2022

Published online: 15 January 2022

\section{References}

1. Koenigsberg RA, Faro S, Chen X, Marlowe F (1996) Nodular fasciitis as a vascular neck mass. Am J Neuroradiol. 17(3):567-569

2. Al Rahbi M, Al-Kindi H, Al-Sheibani S (2015) Post-aural nodular fasciitis. Oman Med J 30(1):63

3. Nishi SP, Brey NV, Sanchez RL (2006) Dermal nodular fasciitis: three case reports of the head and neck and literature review. J Cutan Pathol. 33(5):378-382

4. Singh S, Paul S, Dhall K, Khichy S (2013) Nodular fasciitis: a diagnostic challenge. Indian J Pathol Microbiol. 56(3):288

5. Goldblum JR, Folpe AL, Weiss SW (2014) Enzinger and Weiss Soft tissue tumors, 6th edn. Elsevier, Saunders

6. Fabre D, Fadel E, Singhal S, de Montpreville V, Mussot S, Mercier O, Chataigner O, Dartevelle PG (2009) Complete resection of pulmonary inflammatory pseudotumors has excellent long-term prognosis. J Thorac Cardiovasc Surg 137(2):435-440

7. Cyriac MJ, Celine MI, Kurien G, Puthiode U (2004) Nodular fasciitis. Indian J Dermatol Venereol Leprol 70(4):239-241

\section{Publisher's Note}

Springer Nature remains neutral with regard to jurisdictional claims in published maps and institutional affiliations. 